\title{
Schedule-induced polydipsia: Interactions with wheel running
}

\author{
CORA LEE WETHERINGTON \\ University of North Carolina at Charlotte, Charlotte, North Carolina
}

and

\author{
ANTHONY L. RILEY \\ The American University, Washington, D.C.
}

\begin{abstract}
The effect of wheel running on the level and temporal distribution of schedule-induced polydipsia was examined in 12 food-deprived rats given one food pellet per minute for $1 \mathrm{~h}$. For all rats, wheel running decreased the level of polydipsic intake. It also decreased licking in all six of the 10-sec postpellet intervals except the first. The degree of reduction in licking was a U-shaped function of the 10-sec postpellet intervals and was generally inversely related to the temporal distribution of running. The temporal distribution data are partially consistent with a temporal competition view of the interactions between schedule-induced polydipsia and wheel running.
\end{abstract}

A number of behaviors have been reported to occur in rats under schedules of intermittent food delivery (for reviews, see Falk, 1971; Roper, 1981; Staddon, 1977; Wetherington, 1982). Some of these behaviors (e.g., drinking) appear to be induced by the food schedule (Falk, 1961); others (e.g., wheel running) appear to be noninduced, and appear instead to be simply modulated by the food schedule (Penney \& Schull, 1977; Roper, 1981; Staddon \& Ayres, 1975; Wetherington, Brownstein, \& Shull, 1977). Although behaviors such as drinking and wheel running appear to belong to different functional classes of behavior, that is, induced and noninduced, respectively (Penney \& Schull, 1977; Riley, Wetherington, Delamater, Peele, \& Dacanay, 1985; Staddon, 1977), they do affect each other's respective occurrence when they are concurrently available during spaced food deliveries. For example, as early as 1969 , Segal demonstrated that schedule-induced polydipsia (SIP) was markedly decreased when rats were given concurrent access to a running wheel (see also Riley, Peele, Richard, \& Kulkosky, 1981). Similarly, Roper (1978) reported that SIP increased in rats when the opportunity to run was removed from a baseline condition in which both behaviors were available (see Riley et al., 1981).

Although wheel running clearly can decrease the overall level of SIP, the means by which this decrease is effected at the temporal level has not been systematically examined. Roper (1978) has presented some relevant preliminary data from 2 animals. In Roper's report, the

This research was supported in part by funds from National Science Foundation Grant No. BNS-8406445 and a Reassignment of Duties Leave awarded by the College of Arts and Sciences, University of North Carolina at Charlotte. Reprints may be obtained from Cora Lee Wetherington, Department of Psychology, University of North Carolina at Charlotte, Charlotte, NC 28223, or from Anthony L. Riley, Department of Psychology, The American University, 4400 Massachusetts Avenue, N.W., Washington, DC 20016. temporal distribution of SIP was examined under two conditions of spaced food delivery. Under the first condition, the rats were given the opportunity to both drink and run during the spaced food deliveries. Under the second condition, the rats were given the opportunity only to drink; that is, running was not permitted. When running was prevented, drinking increased at temporal locations formerly occupied by running. Although Roper interpreted this finding in terms of temporal competition between the two behaviors, he did note that drinking also increased in intervals (specifically in the period immediately following pellet delivery) in which little running had previously occurred, indicating that a factor other than temporal competition may also have been operating.

Given that Roper's (1978) data were from only 2 animals, and from a single session when running was permitted and a single session when it was not, more data are necessary in order to characterize the effect of wheel running on the temporal location of drinking. In addition, in Roper's procedure removal of the opportunity to run was accompanied by the removal of the opportunity to gnaw a wood block, thus preventing assessment of the separate contributions of the effects of running and gnawing on drinking. In the present paper, we present temporal distribution data for 12 rats that received alternating sessions in which they were given either concurrent access to a drinking tube and a running wheel or access to a drinking tube alone for a total of 12 sessions.

\section{METHOD}

\footnotetext{
Subjects and Apparatus

The subjects were 12 experimentally naive female rats of LongEvans descent, approximately 90 days old. They were housed in individual wire-mesh cages. The housing quarters were maintained on a 12-h light/12-h dark cycle (lights on at $0800 \mathrm{~h}$ ) at an ambient temperature of $23^{\circ}-24^{\circ} \mathrm{C}$.
} 
The experimental apparatus was a running wheel, $60 \mathrm{~cm}$ in diameter and $9 \mathrm{~cm}$ wide, with a small $11.5 \times 5.5 \times 15.5 \mathrm{~cm}$ access chamber attached to its lower front. The back wall of the wheel was constructed of $1-\mathrm{mm}$-thick aluminum. The front wall and the access chamber were constructed of 3-mm-thick Plexiglas; the floor of the wheel and access chamber was made of 6-mm hardware cloth. A food tray was centered on the door of the access chamber, $3.5 \mathrm{~cm}$ above the floor. A No. $182924-\mathrm{V}$ dc lamp was centered directly above the food tray. The stainless steel spout of a 50-ml graduated Nalgene watering tube, held in place by a ringstand positioned outside the chamber, was flush with a hole in the front wall of the access chamber, $3.4 \mathrm{~cm}$ to the left of the food tray and $3 \mathrm{~cm}$ above the floor.

A torque of approximately $6.0 \mathrm{~J}$ applied in either direction was sufficient to initiate rotation of the wheel. A wheel run was defined by the deflection of a microswitch by any one of eight equally spaced notches in the axle of the wheel. Licks to the drinking spout were recorded via a Grayson-Stadler drinkometer (Model No. 58008). All programming and data recording were accomplished by standard electromechanical devices located in a room adjacent to the one containing the running wheel.

\section{Procedure}

Phase 1: Water alone. Following food deprivation and reduction to $85 \%$ of ad-lib body weight, the rats were given daily sessions in which a single $45-\mathrm{mg}$ Noyes food pellet was delivered independently of their behavior once every minute (a fixed-time [FT] 1-min schedule) for a total of 30 pellet deliveries. Water was continuously available during each session. The opportunity to run, however, was prevented by locking the running wheel. This phase was conducted for 21 consecutive days.

Phase 2: Water and wheel. The procedure for this phase was identical to that of Phase 1 except that the wheel was unlocked, thus providing the opportunity to run. This phase was conducted for 11 consecutive days.

Phase 3: Alternation of availability of running. The procedure for this phase consisted of daily alternation of the procedures of Phases 1 and 2, so that on even-numbered days the rats had concurrent access to a drinking tube and a running wheel, and on oddnumbered days they had access to the drinking tube alone. This alternation procedure lasted until the rats had received six exposures to each condition.

For all phases, after each pellet delivery, licks and/or wheel runs (depending upon the phase in effect) were accumulated in six consecutive 10 -sec intervals corresponding to sixths of the interfood interval.

\section{RESULTS}

Table 1 gives the water intake data (in milliliters) for each rat for each phase of the experiment. The first two columns provide the mean water intake for the last 5 days of Phases 1 and 2, respectively. In Phase 1, when water alone was available, water intake ranged from 7 to $23 \mathrm{ml}$ and averaged $15 \mathrm{ml}$. For all rats except Rat 9 the opportunity to run in Phase 2 resulted in a reduction in water intake. The reduction ranged from $15 \%$ for Rat 7 and Rat 11 to $38 \%$ for Rat 5 (see Phase 2, Table 1). The average reduction for all rats was $27 \%$. In Phase 3, when the opportunity to run alternated on daily sessions, all rats consumed less water when running was permitted. The reduction in water intake ranged from $8 \%$ for Rat 9 to $46 \%$ for Rat 2 and averaged $22 \%$ across all rats.
Table 1

Amount Consumed (in Milliliters) Averaged Over the Last 5 Days of Phases 1 and 2 and All Sessions of Phase 3

\begin{tabular}{lcccc}
\hline Subject & $\begin{array}{c}\text { Phase 1 } \\
\text { Water Alone }\end{array}$ & $\begin{array}{c}\text { Phase 2 } \\
\text { Wheel }\end{array}$ & $\begin{array}{c}\text { Phase 3 } \\
\text { Water Alone }\end{array}$ & $\begin{array}{c}\text { Phase 3 } \\
\text { Wheel }\end{array}$ \\
\hline Rat 1 & 15 & 12 & 14 & 11 \\
Rat 2 & 14 & 9 & 13 & 7 \\
Rat 3 & 23 & 15 & 15 & 13 \\
Rat 4 & 17 & 11 & 13 & 10 \\
Rat 5 & 16 & 10 & 11 & 6 \\
Rat 6 & 19 & 12 & 13 & 10 \\
Rat 7 & 13 & 11 & 11 & 9 \\
Rat 8 & 13 & 9 & 9 & 8 \\
Rat 9 & 7 & 7 & 12 & 11 \\
Rat 10 & 13 & 9 & 8 & 6 \\
Rat 11 & 13 & 11 & 9 & 8 \\
Rat 12 & 16 & 11 & 11 & 9 \\
\hline
\end{tabular}

Figure 1 presents the mean number of licks and wheel revolutions in each 10-sec interval following pellet delivery during Phase 3 . When running was prevented, licking was maximal in the first 10 -sec interval for 8 rats and in the second 10-sec interval for the remaining rats (Rats $1,7,9$, and 11). Thereafter, the frequency of licking sharply declined. When running was permitted, the temporal distribution was clearly affected in all 12 rats. In the second 10 -sec interval, there was a marked decrease in licking. In the remaining intervals, there was also a decrease, although it is somewhat difficult to discern in Figure 1 because the level of licking was generally low even when running was not permitted. During the first 10 -sec interval, licking decreased for only 2 animals (Rats 5 and 12). The remaining rats either increased licking (Rats 1, 2, $3,4,7,9$, and 11 ) or showed no clear change (Rats 6 , 8 , and 10 ).

The distributions of running in Phase 3 exhibited several remarkable regularities. For all the rats, running was low in the first interval, increased during the second, and was maximal in the third (except for Rat 1 , for which running was approximately equal in the third and fourth intervals). Thereafter, running decreased, but was maintained at a level higher than in the first interval.

Although Figure 1 illustrates how the absolute level of licking during the interfood interval was affected by running, it does not depict the relative changes that occurred. Figure 2, therefore, shows for each 10-sec interval a ratio in which the number of licks occurring when running was permitted is expressed as a proportion of the number of licks occurring when running was prevented. Ratios lower than 1.00 indicate that licking was decreased by the opportunity to run. Note that ratios were not computed for cases in which fewer than three licks per 10-sec interval occurred in either the numerator or the denominator; this was the case for the fourth through sixth 10-sec intervals for Rats 8 and 12 . The general shape of these functions is U-shaped, with maximal reductions in licking occurring in either the third or fourth 10 -sec interval. The actual ratio values of the interval in which licking was maximally reduced in Rats 1-12, respectively, are as follows: 


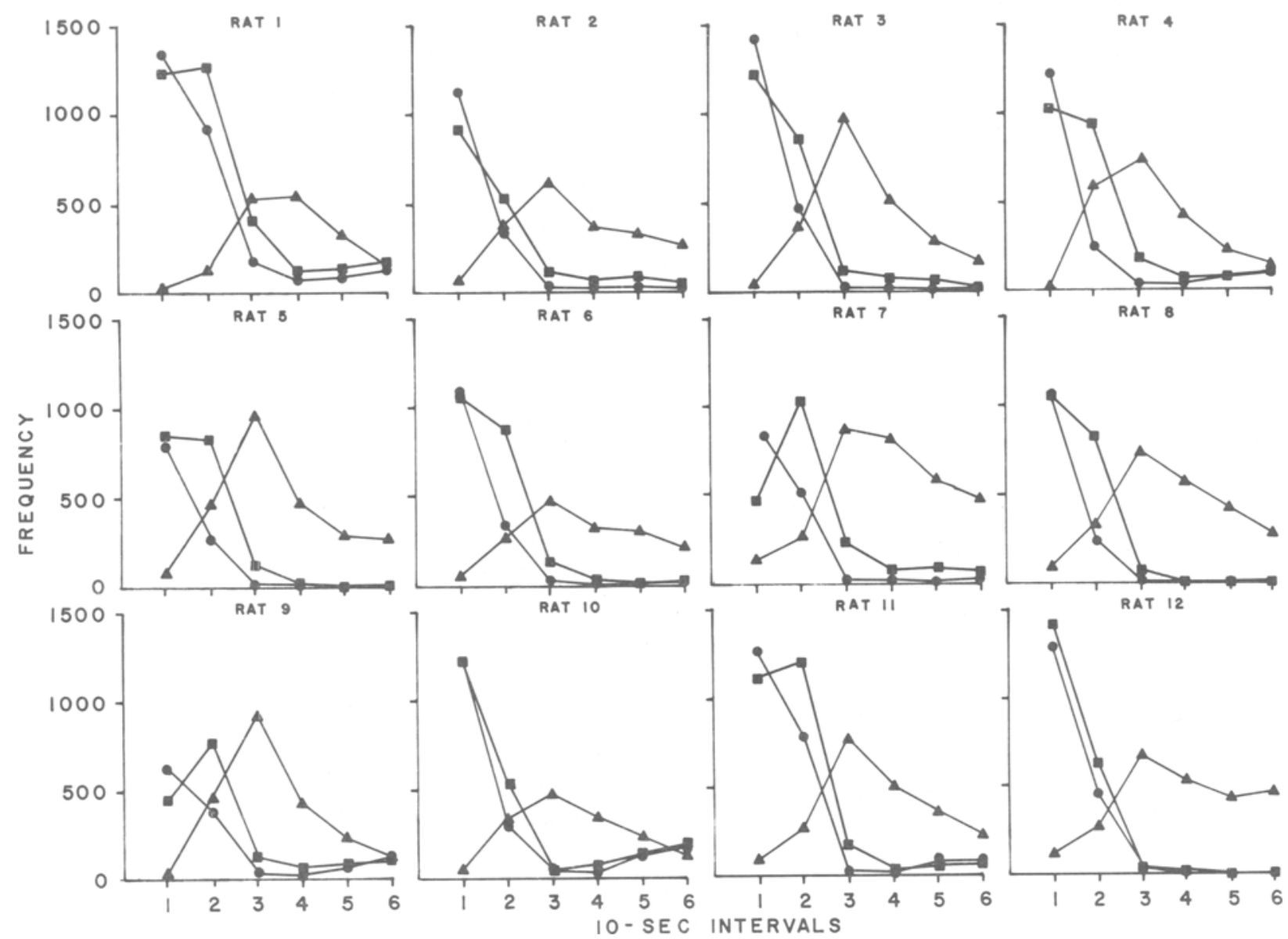

Figure 1. Mean number of licks and runs in the six successive 10-sec intervals following pellet delivery for each rat during Phase 3. Licks during sessions in which running was not permitted are indicated by squares, and licks during sessions in which running was permitted are indicated by circles. Runs are indicated by triangles.

$0.44,0.18,0.12,0.12,0.05,0.18,0.04,0.04,0.24$ $0.49,0.11$, and 0.05 .

Comparison of the functions in Figure 2 with the inverted U-shaped functions for running in Figure 1 reveals that in the first three intervals, as the frequency of running increased, the ratio decreased, indicating less licking. In the last three intervals, as the frequency of running decreased, the ratio increased, indicating more licking.

\section{DISCUSSION}

Although all subjects in the present experiment drank under the schedule of free food deliveries, when running was permitted the overall level of polydipsic licking decreased. This reduction was evident in 11 of the 12 rats when the opportunity to run was offered in separate phases (compare Phases 1 and 2 in Table 1), and in 12 of 12 rats when running was permitted during alternate sessions within the same phase (see Phase 3, Table 1). These data corroborate similar findings reported by Riley et al. (1981), Roper (1978), and Segal (1969).
In addition to its effect on the overall level of licking, wheel running was also examined for its effect on the postpellet temporal distribution of SIP. As described, when the opportunity to run was made available, licking was reduced in the second through sixth 10 -sec intervals following pellet delivery. In fact, changes in the postpellet pattern of licking were closely related to changes in the frequency of running; that is, as running increased, licking decreased, with maximal reductions in licking during intervals in which running was at its maximum. This relationship between running and licking is consistent with the temporal competition account of the interaction of SIP and wheel running previously reported by Roper (1978).

Although the present data are generally consistent with a temporal competition view, there is one discrepant characteristic. Specifically, according to the temporal competition view, all subjects should have displayed decreased licking in any interval in which running occurred concurrently with the availability of water. As described, although all 12 rats displayed some degree of running in the first 10 -sec interval following pellet delivery, a decrease in licking occurred in only 2 subjects. 


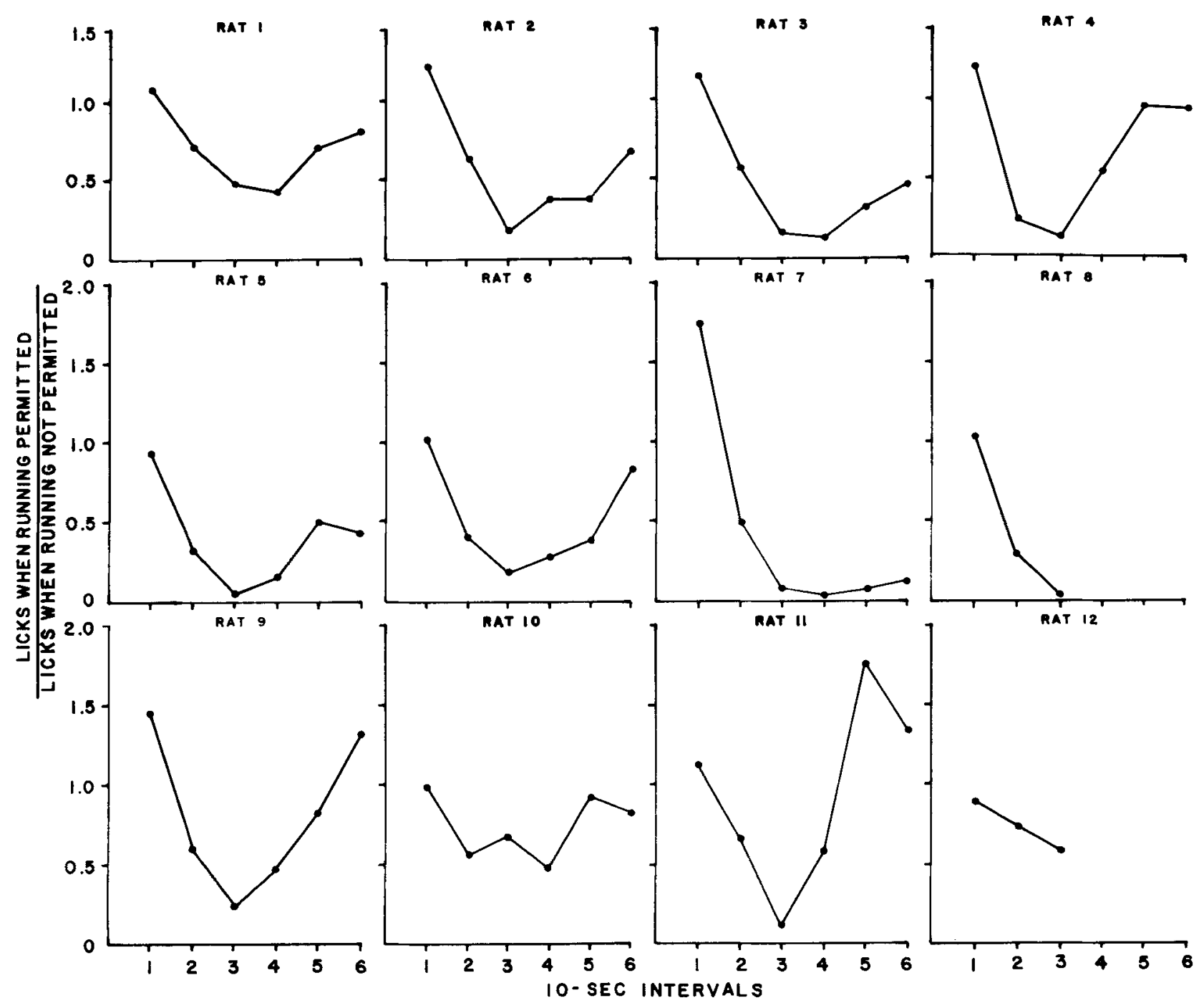

Figure 2. Licking data shown in Figure 1 converted to a ratio by dividing the number of licks occurring when running was permitted by the number of licks occurring when running was not permitted.

The remaining subjects displayed either no change in licking or an actual increase in its frequency. Although inconsistent with the temporal competition of running and licking, the failure of wheel running to compete with licking in this specific interval is actually consistent with other work in SIP in which, under certain circumstances, rats defend a particular volume of water either by changing the local rate of licking or by changing the time spent licking (Flory \& O' Boyle, 1972; Freed \& Mendelson, 1977; Gilbert, 1974; but see Porter, 1985; Wetherington, Lawler, \& Blanco, 1983; Wetherington \& Ware, 1981, for limitations). For example, Flory and O'Boyle (1972) and Gilbert (1974) found that when water was made available only in particular segments of the interfood interval, local increases in licking occurred, so that the overall water intake was virtually unaffected. Although in the present study neither water intake nor licks were con- served when running was permitted (compare amounts consumed when running was permitted and prevented), the operation of a volume-constancy mechanism cannot be ruled out, since the increase in licking in the first 10sec interval exhibited by some rats could simply reflect an unsuccessful attempt at volume constancy.

As described, wheel running and drinking have been regarded as representing two different classes of behavior, noninduced and induced, respectively. One differentiating characteristic between induced and noninduced behaviors is the degree of priority when the opportunity to perform each of these two classes of behavior is given (see Staddon, 1977). Under such conditions, scheduleinduced behaviors are assumed to have priority. In fact, Staddon has suggested that noninduced behaviors, such as wheel running, will be displayed only in the absence of schedule-induced behaviors. From the present analy- 
sis of the overall and temporal interaction of licking and running, it is clear that induced behavior does not necessarily have priority over noninduced behavior (see also Riley et al., 1981; Roper, 1978; Segal, 1969). These data support the growing evidence that induced and noninduced behaviors may have no distinguishing characteristics other than induction (see Killeen, 1975; Roper \& Crossland, 1982; Roper, Edwards, \& Crossland, 1983; Wetherington, 1982).

\section{REFERENCES}

FALK, J. L. (1961). Production of polydipsia in normal rats by an intermittent food schedule. Science, 133, 195-196.

FALK, J. L. (1971). The nature and determinants of adjunctive behavior. Physiology \& Behavior, 6, 577-588.

FLORY, R. K., O'Boyle, M. K. (1972). The effect of limited water availability on schedule-induced polydipsia. Physiology \& Behavior, 8, 147-149.

Freed, W. J., \& Mendelson, J. (1977). Water-intake volume regulation in the rat: Schedule-induced drinking compared with waterdeprivation-induced drinking. Joumal of Comparative \& Physiological Psychology, 91, 564-573.

GILBERT, R. M. (1974). Ubiquity of schedule-induced polydipsia. Journal of the Experimental Analysis of Behavior, 21, 277-284.

KILlEen, P. (1975). On the temporal control of behavior. Psychological Review, 82, 89-115.

PENNeY, J., \& SCHULl, J. (1977). Functional differentiation of adjunctive drinking and wheel running in rats. Animal Learning \& Behavior, 5, 272-280.

PORTER, J. H. (1985). Schedule-induced polydipsia: Another look at water-intake volume regulation. Physiology \& Behavior, 35, 221-227.

Riley, A. L., Peele, D. B., Richard, K. D., \& Kulkosky, P. J. (1981). The interaction of conditioned taste aversions and scheduleinduced polydipsia: Availability of alternative behaviors. Animal Leaming \& Behavior, 9, 287-290.

Riley, A. L., Wetherington, C. L., Delamater, A. R., Peele, D. B., Dacanay, R. J. (1985). The effects of variations in the interpellet interval on wheel running in the rat. Animal Learning \& Behavior, 13, 201-206.
RoPER, T. J. (1978). Diversity and substitutability of adjunctive activities under fixed-interval schedules of food reinforcement. Journal of the Experimental Analysis of Behavior, 30, 83-96.

ROPER, T. J. (1981). What is meant by the term "schedule-induced," and how general is schedule induction? Animal Learning \& Behavior, 9, 433-440.

Roper, T. J., \& Crossland, G. (1982). Schedule-induced wood-chewing in rats and its dependence on body weight. Animal Learning \& $\mathrm{Be}$ havior, 10, 65-71.

Roper, T. J., EdWards, L., \& Crossland, G. (1983). Factors affecting schedule-induced wood chewing in rats: Percentage and rate of reinforcement, and operant requirement. Animal Learming \& Behavior, 11, 35-43.

SEGAL, E. F. (1969). The interaction of psychogenic polydipsia with wheel running in rats. Psychonomic Science, 14, 141-144.

Staddon, J. E. R. (1977). Schedule-induced behavior. In W. K. Honig \& J. E. R. Staddon (Eds.), Handbook of operant behavior (pp. 125152). Englewood Cliffs, NJ: Prentice-Hall.

StAdDon, J. E. R., \& AYres, S. L. (1975). Sequential and temporal properties of behavior induced by a schedule of periodic food delivery. Behaviour, 54, 26-49.

Wetherington, C. L. (1982). Is adjunctive behavior a third class of behavior? Neuroscience \& Biobehavioral Reviews, 6, 329-350.

Wetherington, C. L., Brownstein, A. J., \& Shull, R. L. (1977). Schedule-induced running and chamber size. The Psychological Record, 27, 703-713.

Wetherington, C. L., Lawler, C. P., \& Blanco, I. (1983). Scheduleinduced polydipsia and size of water dipper. Physiology \& Behavior, 30, 669-673.

WETHERINGTON, C. L., \& WARE, R. W. (1981). Schedule-induced polydipsia and mode of access to water. Behaviour Analysis Letters, 1 , 187-198.

(Manuscript received November 14, 1985; revision accepted for publication July 22,1986 .) 